\title{
Direct Application of UNIFAC Activity Coefficient Computer Programs to the Calculation of Solvent Activitles and $\chi$-Parameters for Polymer Solutions
}

\begin{abstract}
Application of UNIFAC computer calculations to polymer solutions does not seem to make sense because of the value of the solvent activity: close to 1.000 over a considerable range of concentrations (up to $90 \%$ of polymer). A simple procedure is proposed to calculate solvent activity coefficients, and thus $\chi$-parameters, such that the easily available UNIFAC computer programs may be applied directly, without any modification.
\end{abstract}

The UNIFAC computer programs published in the book by Fredenslund et al. (1977) readily calculate theoretical solvent activity coefficients $\gamma_{1}$ for solutions of low molecular weight compounds. They do not seem to apply to polymer solutions, as solvent mole fractions $x_{1}$ all close to 1.0 for the whole range of polymer concentrations (up to even $90 \%$ ) have to be input into the programs. Thus, Oishi and Prausnitz (1978) modified the UNIFAC equations to yield activities rather than activity coefficients. Apparently, they did not apply the computer programs published in the book to their equations.

Still, these easily available UNIFAC programs can be applied to polymer solutions, and hence provide a useful base for the estimation of the principal tool of polymer solution studies: the $\chi$-parameter. What one has to do (an example, and the proof of the proposed procedure will be given below) is the following.

1. Input as solute mole fraction $P x_{2}$, where $P=$ degree of polymerization and where $x_{2}=1-x_{1}$, with the solvent mole fraction $x_{1}$ calculated as

$$
x_{1}=\frac{w_{1} M_{2}}{w_{1} M_{2}+w_{2} M_{1}}
$$

(where $w_{1}$ and $w_{2}$ are the solvent and the polymer weight fractions, respectively, and $M_{1}$ and $M_{2}$ the solvent and polymer molecular weights. Calculate $x_{1}$ to 4 figures after the decimal point).

2. Calculate the solvent activity $\ln a_{1}$ (more precise: the sum of its combinatorial and its residual part, $\ln a_{1}=\ln$ $a_{1}{ }^{\mathrm{C}}+\ln a_{1}{ }^{\mathrm{R}}$ ) from $\gamma_{1}$, output by the computer program as

$$
\ln a_{1}=\ln \gamma_{1}+\phi_{2}^{\prime} \frac{r_{1}}{r_{2}}+\ln x_{1}
$$

where

$$
\phi_{2}{ }^{\prime}=\frac{P x_{2} r_{2}}{x_{1} r_{1}+P x_{2} r_{2}}
$$

and where $r_{1}$ and $r_{2}$ are the molar volume parameters of solvent and monomeric unit, respectively, calculated from numbers $\left(\nu_{k}{ }^{(i)}\right)$ and molar volumes $R_{k}$ of groups in the usual way from $r_{i}=\sum_{k} \nu_{k}{ }^{(i)} R_{k}$ (see pages 31 and 32 of the book by Fredenslund et al. (1977)).

Thus, from the numerical data of Oishi and Prausnitz (1978) $\left(w_{1}=0.09575, M_{1}=78.11\right.$, and $\left.M_{2}=4 \times 10^{4}\right)$ for the system benzene-polyisobutylene we can calculate: $x_{1}$ $=0.9819$. Using for the monomer molecular weight $m$ the value of 56.10 , we find $P=M_{2} / m=713.01$ and $P x_{2}=$ 12.8931. The computer program calculates (using the functional groups 10 (six times) for the solvent and 1 (twice), 2 (once), 4 (once) for the monomeric unit) $\gamma_{1}=$ 0.1046 . (The group numbers refer to the groups tabulated by Gmehling et al., 1982).

Oishi and Prausnitz (1978) calculated using these groups $r_{1}^{\prime}$ and $r_{2}^{\prime}$, which are equal to $r_{1} / M_{1}$ and $r_{2} / m$, respectively. Using their $r_{1}^{\prime}$ and $r_{2}^{\prime}$, and the above-mentioned values for $m, M_{1}, x_{1}$ and $P x_{2}$, we find

$$
\begin{gathered}
\phi_{2}{ }^{\prime}\left(r_{1} / r_{2}\right)=[(12.8931 \times 0.04806 \times 56.10) /(0.9819 \times \\
0.04081 \times 78.11+12.8931 \times 0.04806 \times 56.10)] \times \\
{[(0.04081 \times 78.11) /(0.04806 \times 56.10)]=}
\end{gathered}
$$$$
0.917 \times 1.1823=1.084
$$

Then

$$
\ln a_{1}=\ln 0.1046+1.084+\ln 0.9819=-1.192
$$

which is, in view of the many rounding off errors involved in the procedures for calculation of $\gamma_{1}, \phi_{2}{ }^{\prime}$, and $x_{1}$, satisfactorily in agreement with Oishi and Prausnitz' (1978) result

$$
\ln a_{1}=\ln a_{1}{ }^{\mathrm{C}}+\ln a_{1}{ }^{\mathrm{R}}=-1.53+0.336=-1.194
$$

The proof of this procedure rests upon inspection of the equation derived for $\ln a_{1}$ by Oishi and Prausnitz (1978)

$$
\begin{aligned}
\ln a_{1}= & \ln \phi_{1}{ }^{\prime}+\phi_{2}{ }^{\prime}+\frac{z}{2} M_{1} q_{1}{ }^{\prime} \ln \frac{\theta_{1}{ }^{\prime}}{{\phi_{1}}^{\prime}}- \\
& \frac{z}{2} M_{1} q_{1}{ }^{\prime}\left(1-\frac{\phi_{1}{ }^{\prime}}{\theta_{1}{ }^{\prime}}\right)+\sum_{k} \nu_{k}{ }^{(i)}\left[\ln \Gamma_{k}-\ln \Gamma_{k}{ }^{(i)}\right]
\end{aligned}
$$

(see their eq 5 and 9) and that of the book by Fredenslund et al. (1977)

$$
\begin{aligned}
\ln \gamma_{1}=\ln \frac{\phi_{1}}{x_{1}}+ & \frac{z}{2} q_{1} \ln \frac{\theta_{1}}{\phi_{1}}-\frac{z}{2} q_{1}\left(1-\frac{\phi_{1}}{\theta_{1}}\right)+ \\
& \phi_{2}\left(1-\frac{r_{1}}{r_{2}}\right)+\sum \nu_{k}^{(i)}\left[\ln \Gamma_{k}-\ln \Gamma_{k}^{(i)}\right]
\end{aligned}
$$

The latter equation results, after some rearrangement, from the eq 4.7 and 4.9 of the book (p 31), into which the pertinent definitions of $r_{1}, r_{2}, q_{1}, q_{2}, \phi_{1}, \phi_{2}, \theta_{1}$, and $\theta_{2}$ have been substituted. (For these and other symbols see the literature cited).

Equation 3 for $\phi_{2}{ }^{\prime}$ follows directly from the Oishi and Prausnitz (1978) definition of $\phi_{i}{ }^{\prime}$, together with their definitions of $r_{i}{ }^{\prime}$. A like result follows for $\phi_{1}{ }^{\prime}, \theta_{1}{ }^{\prime}$, and $\theta_{2}{ }^{\prime}$. Thus, equations like (3) should be substituted for $\phi_{1}, \phi_{2}$, $\theta_{1}$, and $\theta_{2}$ in eq 5 , the basic equation of the UNIFAC computer program. When this is done, and when the definitions $q_{1}^{\prime}=q_{1} / M_{1}$ and $q_{2}{ }^{\prime}=q_{2} / m$ are applied, our eq 2 results from inspection of eq 4 and 5 .

Oishi and Prausnitz (1978) also have shown that the complete theoretical equation for the solvent activity of a polymer solution should contain a free volume term in addition to the combinatorial and the residual term

$$
\ln a_{1}=\ln a_{1}{ }^{\mathrm{R}}+\ln a_{1}{ }^{\mathrm{C}}+\ln a_{1}{ }^{\mathrm{FV}}
$$

The $\chi$-parameter is defined by Gottlieb and Herskowitz (1981) from

$$
\ln a_{1}=\left(\mu_{1}-\mu_{1}{ }^{0}\right) / R T=\ln {\phi_{1}}^{\prime}+\phi_{2}{ }^{\prime}+\chi\left(\phi_{2}{ }^{\prime}\right)^{2}
$$

and the sum $\ln a_{1}{ }^{\mathrm{R}}+\ln {a_{1}}^{\mathrm{C}}$ is calculated by the procedure mentioned above. Thus we get finally for the complete equation for $\chi$ 


$$
\begin{array}{r}
\chi=\left\{\left(\ln \gamma_{1}\right)_{\text {comp }}+\ln x_{1}+\phi_{2}{ }^{\prime}\left(r_{1}{ }^{\prime} M_{1}\right) /\left(r_{2}{ }^{\prime} m\right)-\ln \phi_{1}{ }^{\prime}-\right. \\
\left.\phi_{2}{ }^{\prime}\right\} /\left(\phi_{2}\right)^{2}+\left(\ln a_{1}{ }^{\mathrm{Fv}}\right) /\left(\phi_{2}{ }^{\prime}\right)^{2}
\end{array}
$$

For $\ln a_{1}{ }^{F V}$ we could use the Oishi and Frausnitz (1978) expression, but also one to be derived from the lattice fluid theory by Sanchez and Lacombe (1978). Elsewhere, it will be shown that it leads to a different numerical value for the $\chi$-parameter. Hence, a satisfactory theoretical prediction of the $\chi$-parameter does not only depend on a proper choice of the group parameters (Prausnitz, 1982) but also on a proper choice of the volume term $a_{1} \mathrm{FV}$. For the study of tendencies in the course of the $\chi$-parameter with $w_{2}$ for a homologous series of solvents for one polymer, eq 8 remains very useful, provided that a consistent set of UNIFAC parameters is used (derived from vapor-liquid data, or from liquid-liquid data, in accordance with the system studied).

The above procedure produces solvent activities that do not depend on polymer molecular weight (the number average $\bar{M}_{n}$, by definition) substituted in eq 1 , provided that $\bar{M}_{\mathrm{n}}$ is "high enough" $\left(\bar{M}_{\mathrm{n}} / M_{1}>100\right.$, say). This follows from the derivation of eq $4:$ the occurrence of the term $\phi_{2}^{\prime}$ makes it typical for polymer solutions. There are no $\bar{M}_{\mathrm{n}}$ dependent terms, except for a coefficient of $\phi_{2}{ }^{\prime}$. It is close to 1.0 , however, if $\bar{M}_{\mathrm{n}}$ is high enough.
Thus, in order to apply the above procedure, choose $\bar{M}_{\mathrm{n}}$ high enough to validate eq 4 (and 7) and low enough to provide a range in $x_{1}$ and $P x_{2}$ that covers a reasonable range in polymer weight fractions $w_{2}$. Due to rounding off errors, there will remain a slight dependence of $\ln a_{1}$ and $\chi$ on $\bar{M}_{\mathrm{n}}$ in the region of low $w_{2}$ values (where $0.9980<x_{1}$ $<0.9999$ ), however.

\section{Literature Cited}

Fredenslund, A.; Gmehling, J.; Rasmussen, P. "Vapor-Liquid Equillbria using Unifac": Elsevier Scientific Publishing Company: Amsterdam-Oxford-Now York, 1977.

Gmehling, J.; Rasmussen, $P_{. ;}$Fredenslund, A. Ind. Eng. Chem. Process Des. Dev. 1982, 21, 118 .

Gottlieb, M.; Herskowitz, M. Macromolecules 1981, 14, 1468.

Oishi, T.; Prausnitz, J. M. Ind. Eng. Chem. Process Des. Dev. 1978, 17 333.

Prausnitz, J. M. Ind. Eng. Chem. Process. Des. Dev. 1982, 21, 537.

Sanchez, I. C.; Lacombe, R. H. Macromolecules 1978, 11, 1145.

Department of Chemical Johannes W. van den Berg Technology

Twente University of Technology

7500 AE Enschede, The Netherlands

Received for review October 3, 1983 Accepted December 5, 1983 